\title{
The Relationship between Ultrawatersoluble LDL (UWS-LDL) and Platelet Aggregability*
}

\author{
Tsugumichi Uemura**, Takemichi KanaZawA**, Masahiro IzawA**, \\ Hirohiko KANEKO**, Kogo ONODERA**, Hirobumi MetoKI***, \\ Yoshito SHIMANAKA***, Reiko KAWAHARA*** and Yasaburo OIKE***
}

Many reports have described that the serum low density lipoprotein (LDL) plays an important role ${ }^{1-3)}$ in the pathophysiology of human atherosclerotic diseases.

Kanazawa et al. have found ${ }^{4)}$ that native LDL can be separated into two fractions, ultrawatersoluble LDL (UWS-LDL) and non-watersoluble LDL (non-WS-LDL) by water dialysis.

They also reported ${ }^{5-7)}$ that the levels of serum UWS-LDL were significantly higher in the patients with arteriosclerotic diseases e.g. ischemic heart disease and cerebral infarction, and that UWSLDL was internalized into the cultured mouse peritoneal macrophages in a greater amount than native LDL.

In this experiment, the effects of these lipoproteins on the platelet aggregability were investigated to find the relationship between the pathogenesis of arteriosclerotic diseases and UWS-LDL.

\section{Experimental Subjects and Methods}

1) Subjects

The subjects were 8 healthy persons and 10 atherosclerotic patients, namely, 6 cerebral vascular disorders, 3 essential hypertensions, and 1 old myocardial infarction.

2) Blood sampling and plasma separation

Twenty-seven $\mathrm{m} l$ of venous blood were drawn into the sterile syringe with $3.8 \%$ sodium citrate $3.0 \mathrm{~m} l$ from each fasting patient early in the morning, and plasma was separated by centrifugation at 3,000 rpm for 20 minutes.

3) Preparation of native LDL and UWS-LDL

The native LDL was separated by ultracentrifu-

* This paper was presented at the 18th meeting of the Japan Atherosclerosis Society in Japan in May 1986

** The Second Department of Internal Medicine, Hirosaki University School of Medicine

*** Reimeikyo Rehabilitation Hospital gation of plasma according to the method of Havel et al. The UWS-LDL was obtained by Kanazawa's method. ${ }^{4)}$ Each native LDL was divided into two groups by the time of water dialysis. One group was dialyzed against flowing water for 48 hours, and the other for 120 hours. Then these were centrifuged at $3,000 \mathrm{rpm}$ for 30 minutes. The supernatant was defined as UWS-LDL, and the precipitate as non-WS-LDL. All of the lipoproteins were kept in a refrigerator after exchange of nitrogen gas.

4) Evaluation of platelet aggregability

Twenty $\mu l$ of native LDL, UWS-LDL or saline was added to $180 \mu l$ of platelet rich plasma (PRP). Then, $20 \mu l$ of relatively low concentration of ADP, collagen, epinephrine was added to those tubes, and the platelet aggregabilities were estimated according to the method of Born. The concentration of ADP, collagen was chosen to keep the maximum platelet aggregability (MPA) below $40 \%$, and that of epinephrine was chosen to keep the time that reached $1 / 2$ of MPA (T $1 / 2)$ markedly extended, because a little aggregability was very easy to decide the effect of adding lipoproteins.

All experiments were performed within 1 hour after separation of PRP. Because platelet aggregability was changeab $\odot$ after 1 hour from separation of PRP.

\section{Experimental Results}

1) The effect of UWS-LDL on platelet aggregation induced by ADP

Figure 1 shows that native LDL inhibited slightly the platelet aggregation induced by ADP (Fig. 1B), but UWS-LDL made it increased markedly (Fig. 1 C, D). As shown in Fig. 2, UWS-LDL dialyzed against flowing water for 120 hours enhanced MPA induced by ADP more than that for 48 hours.

2) The effect of UWS-LDL on platelet aggregation induced by collagen 
動脈硬化 Vol.15 No. 31987

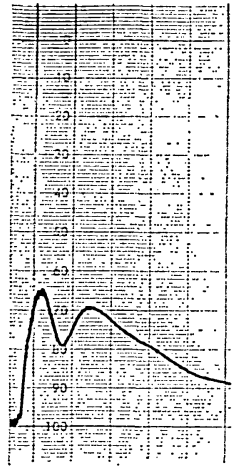

A

PRP $180 \mu \ell$

Saline $20 \mu \mathrm{l}$

ADP $20 \mu \ell$

$(1.0 \mu \mathrm{M} / \ell)$

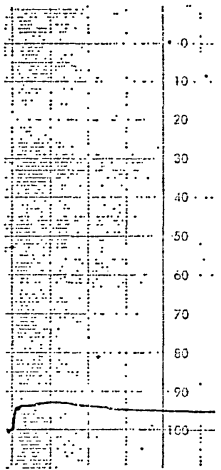

B

$\begin{array}{lr}\text { PRP } & 180 \mu \ell \\ \text { LDL } & 20 \mu \ell \\ \text { ADP } & 20 \mu \ell\end{array}$

$(1.0 \mu \mathrm{M} / \ell)$

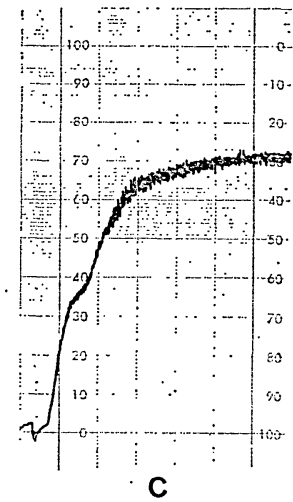

PRP $180 \mu \ell$

UWS-LDL(48h) $20 \mu \ell$

ADP

$20 \mu \ell$

$(1.0 \mu \mathrm{M} / \ell)$

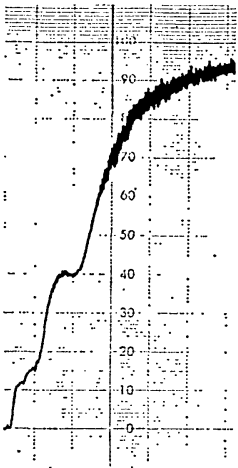

D

PRP $180 \mu \ell$

UWS-LDL(120h) $20 \mu \ell$ ADP $20 \mu \ell$

$(1.0 \mu \mathrm{M} / Q)$

Fig. 1 The influence of LDL and UWS-LDL on platelet aggregation by ADP. (Arteriosclerotic disease)

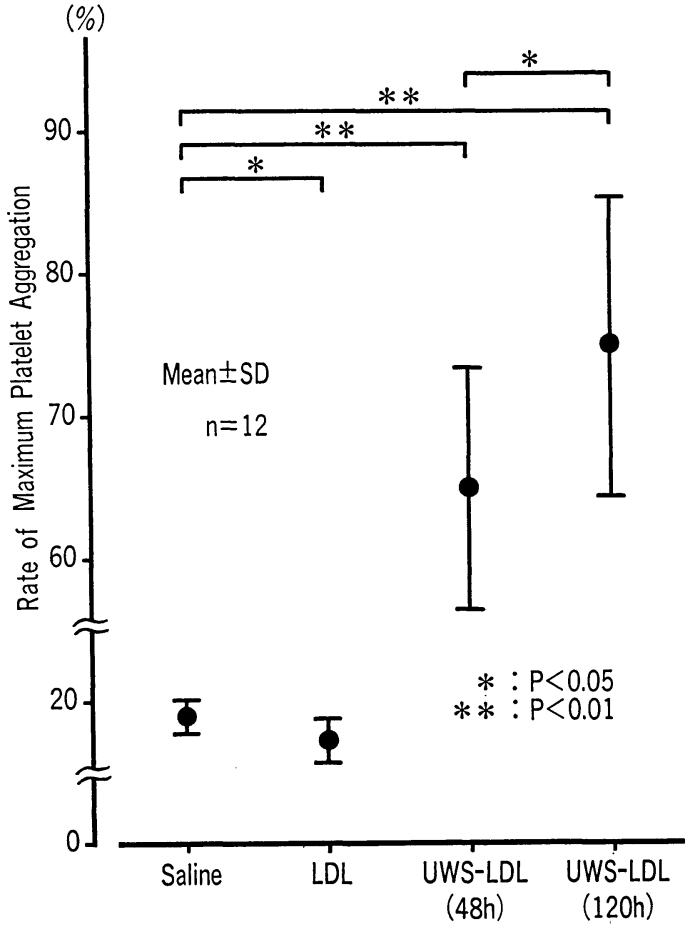

Fig. 2 The influence of LDL and UWS-LDL on platelet aggregation by ADP. 
The Relationship between Ultrawatersoluble LDL (UWS-LDL) and Platelet Aggregability

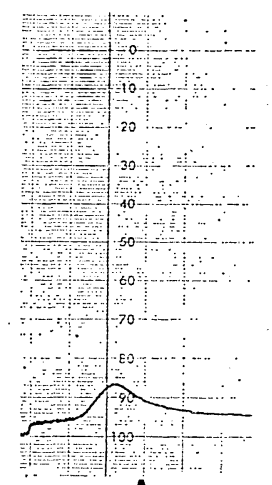

A

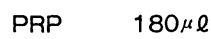

Saline $20 \mu 0$

Collagen $20 \mu \ell$

$(1.0 \mu \mathrm{g} / \mathrm{m} \ell)$

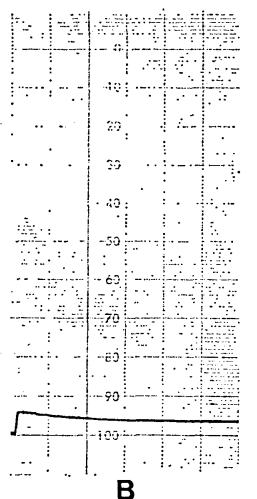

$\mathrm{PRP} \quad 180 \mu \mathrm{l}$

LDL $20 \mu \ell$

Collagen $20 \mu \mathrm{l}$

$(1.0 \mu \mathrm{g} / \mathrm{ml})$
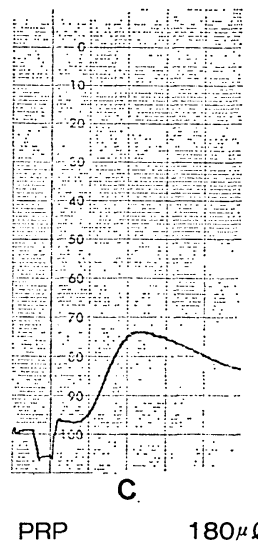

UWS-LDL(48h) $20 \mu l$

Collagen $20 \mu \mathrm{l}$

$(1.0 \mu \mathrm{g} / \mathrm{ml})$

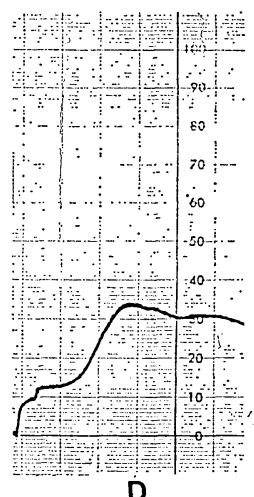

$180 \mu \ell$

UWS-LDL(120h) $20 \mu l$

Collagen $20 \mu \mathrm{l}$

$(1.0 \mu \mathrm{g} / \mathrm{m} \ell)$

Fig. 3 The influence of LDL and UWS-LDL on platelet aggregation by collagen. (Arteriosclerotic disease)

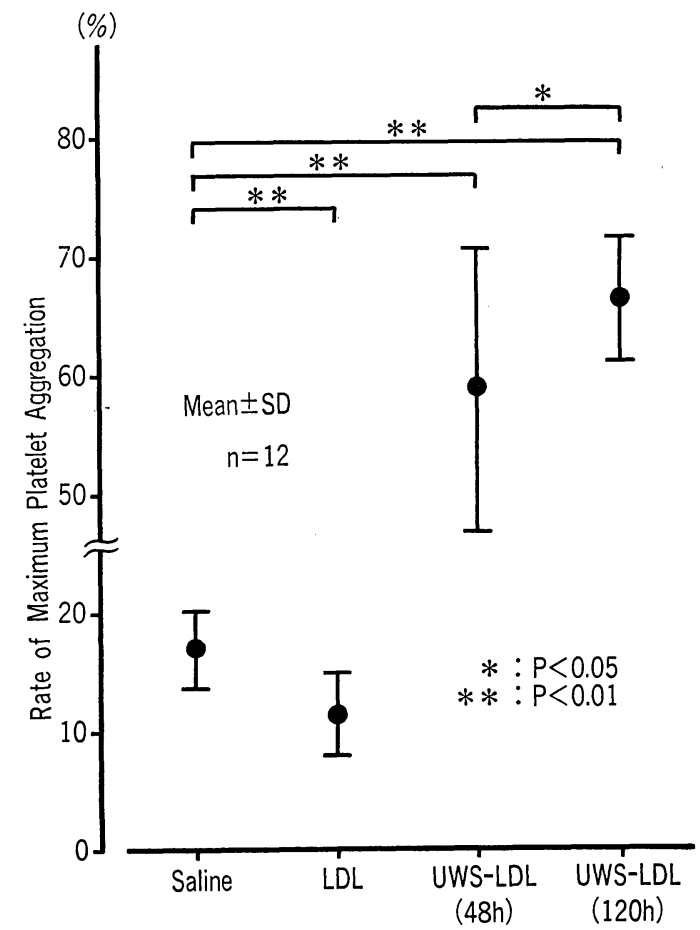

Fig. 4 The influence of LDL and UWS-LDL on platelet aggregation by collagen. 


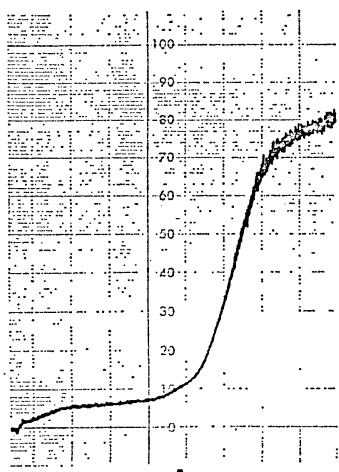

A

PRP $\quad 180 \mu \ell$

Saline $\quad 20 \mu \ell$

Epinephrine $20 \mu \ell$

$(1.0 \mu \mathrm{g} / \mathrm{m \ell})$

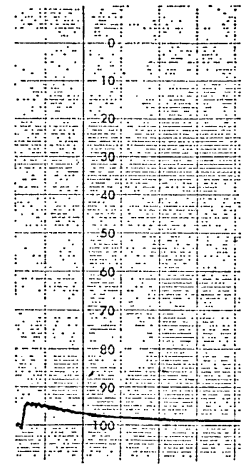

B

PRP $180 \mu \ell$

LDL $\quad 20^{\mu} \ell$

Epinephrine $20 \mu \ell$

$(1.0 \mu \mathrm{g} / \mathrm{m} \ell)$

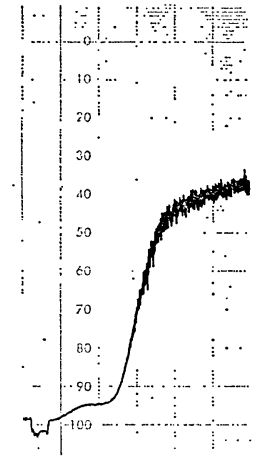

C

PRP

$180 \mu \ell$

UWS-LDL(48h) $20 \mu \ell$

Epinephrine $20 \mu \ell$

$(1.0 \mu \mathrm{g} / \mathrm{m} \ell)$

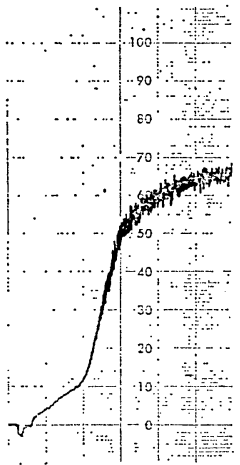

D

$180 \mu \ell$

UWS-LDL(120h) $20 \mu \ell$

Epinephrine $20 \mu \ell$

$(1.0 \mu \mathrm{g} / \mathrm{m \ell})$

Fig. 5 The influence of LDL and UWS-LDL on platelet aggregation by epinephrine. (Arteriosclerotic disease)

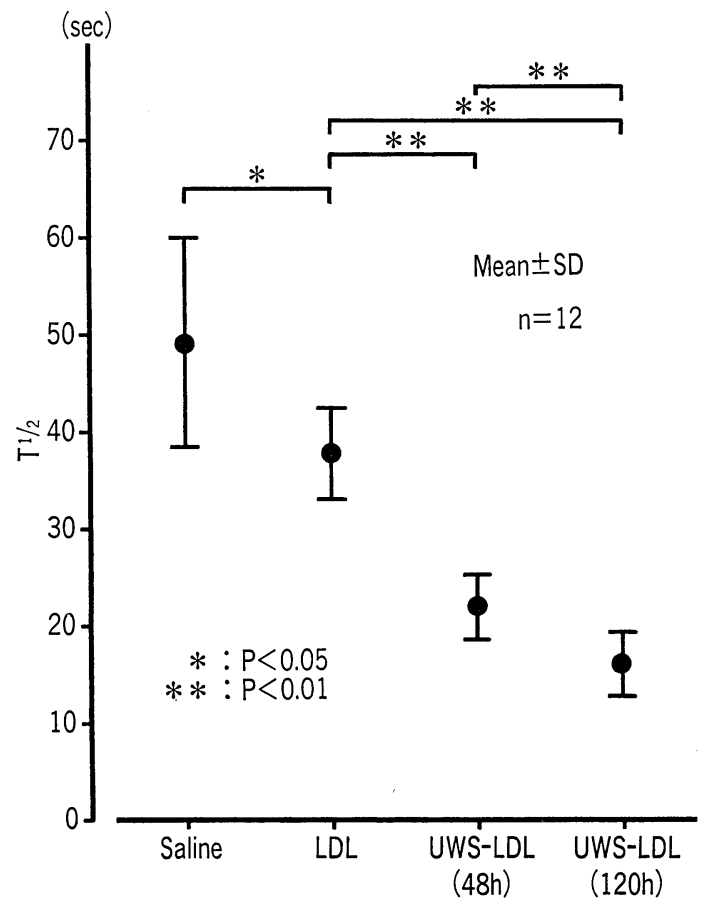

Fig. 6 The influence of LDL and UWS-LDL on platelet aggregation by epinephrine.

Figure 3 shows that native LDL inhibited slightly the platelet aggregation induced by collagen (Fig. 3 B), but UWS-LDL stimulated it (Fig. 3 C, D). Also, as the same way Fig. 4 shows the stimulating effect of UWS-LDL. UWS-LDL dialyzed a- gainst flowing water for 120 hours enhanced MPA induced by collagen more than that for 48 hours.

3) The effect of UWS-LDL on platelet aggregation induced by epinephrine

As shown in Fig. 5, native LDL slightly stimu- 
lated the platelet aggregation induced by epinephrine (Fig. 5 B), whereas UWS-LDL stimulated it markedly (Fig. $5 \mathrm{C}, \mathrm{D}$ ).

And also UWS-LDL dialyzed for 120 hours enhanced MPA more than 48 hours (Fig. 6).

\section{Discussion}

Malondialdehide-LDL, acetylated LDL, and LDL incubated with smooth muscle cells have been reported as modified LDL by Goldstein et al. or Fogelman et al. ${ }^{8,9)}$ These modified LDL was internalized so much into macrophages, accordingly they insisted that these modified LDL plays an important role in the mechanism of arteriosclerosis.

UWS-LDL which Kanazawa et al. also reported is one kind of modified LDLs. They reported this UWS-LDL is very important as well as modified LDL divided by Goldstein or Fogelman. Namely they reported ${ }^{4-7)}$ that the levels of serum UWSLDL were significantly higher in the patients with arteriosclerotic diseases e.g. ischemic heart disease, cerebral infarction and that UWS-LDL was internalized into the cultured mouse peritoneal macrophages in a greater amount than native LDL. ${ }^{10}$ )

It has been reported what many platelets was accumulated in the portion of sclerotic lesion and the pathological changes on the arteries progress so much for growing of mass of platelets.

Therefore, in this experiment we planned to clarify the relationship between platelet aggregation and UWS-lipoprotein.

The results of this experiment will prove the probability that UWS-LDL has a close relation with the initiation and development of atherosclerosis because of the excitation of platelet aggregability.

It is unknown that substance of UWS-LDL stimulate the platelet aggregation.

Kanazawa et al. found that from native LDL, five kinds of lipids, namely, free cholesterol (FC), cholesterol ester (CE), triglyceride (TG), free fatty acid (FFA), phospholipid (PL), can be defined by thin layer chromatography, whereas from UWSLDL furthermore several lipids were detected. They also found that oxygen bubbling to native LDL made it UWS-LDL analogue.

These might suggest that oxidation or peroxidation of native LDL have relationships to the excitability of platelet aggregation.
Furthermore Asano et al. reported ${ }^{11)}$ that unsaturated fatty acid peroxides caused a concentration-dependent contraction of rabbit aortic strip.

The stimulating effect of UWS-LDL on the platelet aggregability is very important to the progression of atherosclerosis because lipids and platelets exist so much in sclerotic tissues.

\section{References}

1) Fogelman, O.: Metabolism of plasma lipoproteins. Acta Med. Scand. (suppl.), 614: 1 (1977).

2) Hamsten, A., et al.: Serum lipoproteins and Apolipoproteins in young male survivors of myocardial infarction. Atherosclerosis, 59: 223 (1986).

3) Bradby, G. H. V., et al.: The binding of total low density lipoproteins in human arterial intima affected and unaffected by atherosclerosis. Atherosclerosis, 32: 403 (1979).

4) Kanazawa, T., et al.: The method of fractionation of ultrawatersoluble and non-watersoluble lipoproteins from serum, and the significances of those lipoproteins in stroke. Jpn. Stroke, 3: 243 (1981).

5) Kanazawa, T., et al.: A Study on ultra-watersoluble lipoprotein. J. Jpn. Atheroscler. Soc., 7: 285 (1979).

6) Kanazawa, T., et al.: Lipids and binding hexoses in lipoprotein fraction by ultracentrifugation, Hirosaki Med. J., 32: 586 (1980).

7) Kanazawa, T., et al.: Relationships between lipids and glycoproteins in arteriosclerosis-Serum binding hexose with lipid and serum binding hexose without lipid in patient with hypertension, diabetes mellitus or calcification of aortic arch. J. Jpn. Atheroscler. Soc., 6: 541 (1979).

8) Goldstein, J. L., et al.: Binding site on macrophages that mediates uptake and degradation of acetylated low density lipoprotein, producing massive cholesterol deposition. Proc. Natl. Acad. Sci., 76: 333 (1979).

9) Fogelman, A. F., et al.: Malondialdehide alteration of low density lipoproteins leads to cholesteryl ester accumulation in human monocyte-macrophages. Proc. Natl. Acad. Sci., 77: 2214 (1980).

10) Kanazawa, T., et al.: Internalization of VLDL and LDL into mouse peritoneal macrophage, VIIth International Symposium on Atherosclerosis, satellite symposium. Cell biology of the artery wall (1985).

11) Asano, M. and Hidaka, H.: Contractile response of isolated rabbit aortic strips to unsaturated fatty acid peroxides. J. Pharmacol. Exp. Therap., 208: 347-353 (1979). 


\section{Summary}

The effects of native LDL and UWS-LDL on the platelet aggregation induced by ADP, collagen, and epinephrine were investigated.

1) Native LDL inhibited slightly the platelet aggregation induced by ADP or collagen, but slightly enhanced it induced by epinephrine.

2) UWS-LDL enhanced markedly the platelet aggregation in these three aggregants.

3) UWS-LDL dialyzed against flowing water for 120 hours enhanced the platelet aggregation more than that for 48 hours.

It was speculated that the interaction between platelet and UWS-LDL plays a very important role in formation and progression of arteriosclerotic lesion.

Key words: Native LDL, UWS-LDL, Platelet aggregability. 